\title{
Panoftalmite tardia após trabeculectomia com mitomicina $\mathrm{C}$
}

\section{Late panophthalmitis after trabeculectomy with mitomycin $C$}

Nikias Alves da Silva1, Danielle Carlier², Felício Aristóteles da Silva ${ }^{3}$

\section{Resumo}

Infecção intraocular é a mais dramática e temível complicação após uma trabeculectomia, com sequelas anatômicas significativas e grave comprometimento funcional, por vezes irreversíveis. Trata-se no presente caso de um paciente do sexo masculino, portador de glaucoma congênito, submetido à trabeculectomia bilateral aos 13 anos de idade, e uma segunda trabeculectomia com mitomicina $\mathrm{C}$ aos 32 anos no olho direito (OD), que se apresentou 8 anos após o procedimento com endoftalmite neste olho. Após tratamento anti-inflamatório e antibioticoterápico, tópico e intravítreo, intensivos, o processo foi controlado, restando um olho hipotrófico e amaurótico com catarata e descolamento de retina totais.

Descritores: Panoftalmite; Trabeculectomia; Mitomicina; Agentes antibacterianos; Relatos de Casos

\footnotetext{
1 Assistente da Clínica de Olhos da Santa Casa de Misericórdia de Belo Horizonte; Médico Oftalmologista do Centro de Oftalmologia Avançada; Pós-graduando nível doutorado do Hospital São Geraldo - HC/UFMG - Belo Horizonte (MG) - Brasil;

${ }^{2}$ Assistente da Clínica de Olhos da Santa Casa de Misericórdia de Belo Horizonte, Belo Horizonte (MG)- Brasil;

3 Professor da Clínica de Olhos da Santa Casa de Misericórdia de Belo Horizonte; Associado do Centro de Oftalmologia Avançada, Belo Horizonte (MG) - Brasil.
}

Trabalho realizado na Clínica de Olhos da Santa Casa de Misericórdia de Belo Horizonte- Belo Horizonte (MG) - Brasil. 


\section{INTRODUÇÃO}

$\mathbf{I}$ nfecções relacionadas à presença de uma ampola filtrante, outrora mais raras $(0,2 \%-1,5 \%),{ }^{1}$ têm sido cada vez mais frequentes com o uso de antimitóticos $(1,0 \%-3,8 \%)^{(1)}$, podendo alcançar até $17 \%{ }^{(2)}$. Os sinais e sintomas são dor ocular, baixa de acuidade visual, fotofobia, congestão ocular, quemose conjuntival e edema palpebral. A infecção pode se restringir à ampola filtrante ("blebitis"), estender-se à câmara anterior (endoftalmite), com reação fibrinopurulenta de intensidade variável e hipópio e, por fim, envolver também o segmento posterior, com turvação do vítreo e repercussão orbitária (panoftalmite). Mais comumente são infecções tardias ${ }^{(3,4)}$, podendo ocorrer até 60 anos após a cirurgia ${ }^{(5)}$. Álém do uso de antimitóticos, outros importantes fatores de risco para a instalação de tais infecções são trabeculectomia inferior ${ }^{(6,7)}$, pacientes jovens, ${ }^{7}$ ampola filtrante de parede fina e avascular, vazamento na ampola filtrante ${ }^{(6,11)}$, más condições higiênicas, episódios de conjuntivite, obstrução lacrimal ${ }^{(11)}$, uso episódico ou contínuo de antibiótico no pós-operatório ${ }^{(12)}$,diabetes mellitus $^{(13)}$, uso de lentes de contato e manipulação da ampola filtrante (agulhamento, sutura compressiva, secção de sutura com laser, revisão cirúrgica e injeção de sangue autólogo $)^{(11)}$. Mesmo quando se consegue debelar o quadro infeccioso e preservar o globo ocular, porque enucleação ou evisceração podem ser necessárias em até $22 \%$ dos casos, frequentemente o funcionamento da ampola filtrante é comprometido em consequência de sua fibrose e desaparecimento, com descompensação da pressão intraocular (PIO) (acima de $21 \mathrm{mmHg}$ em $11 \%)^{(11)}$.

Este caso ilustra a ocorrência tardia de panoftalmite oito anos após a realização de uma trabeculectomia com mitomicina $\mathrm{C}$ em um paciente com glaucoma congênito já submetido a uma cirurgia antiglaucomatosa, bem como o trágico desfecho desta complicação, do ponto de vista funcional, apesar da preservação anatômica do olho.

\section{Apresentação do caso}

JMJ, sexo masculino, 40 anos de idade, natural de e residente em Santa Bárbara, MG, agricultor. Foi atendido em outro serviço aos 21 anos de idade, do qual trouxe cópia do prontuário, onde informou que era portador de glaucoma em ambos os olhos, congênito no OD, diagnosticado aos 3 anos de idade, tendo sido submetido à trabeculectomia nos dois olhos aos 13 anos. Não havia antecedentes familiares de glaucoma e os pais eram pri-

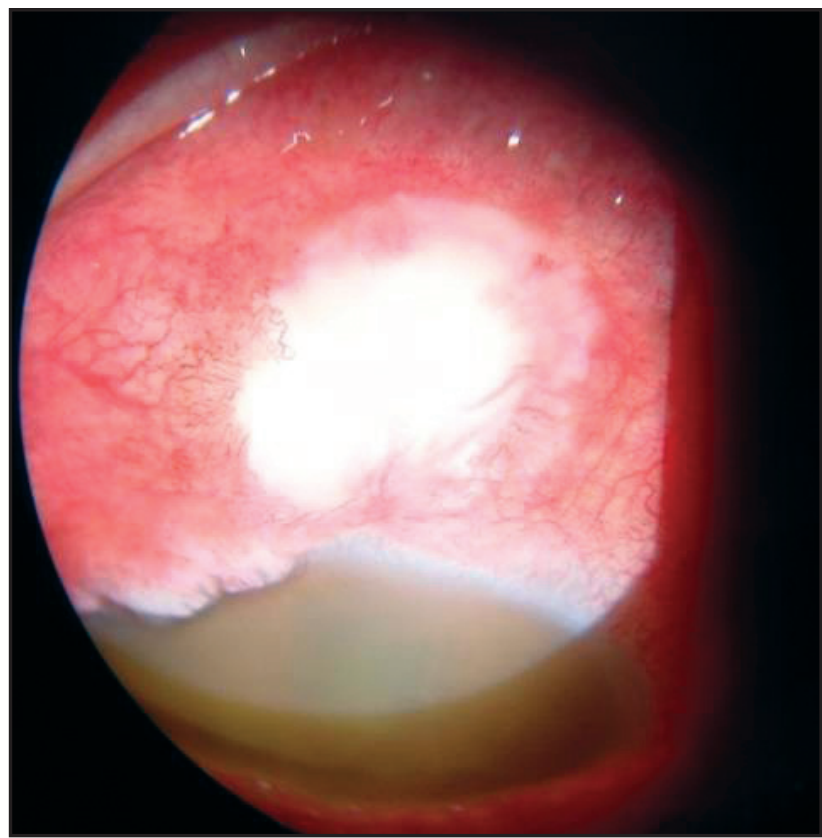

Figura 1: Congestão conjuntival e infiltração purulenta da ampola filtrante

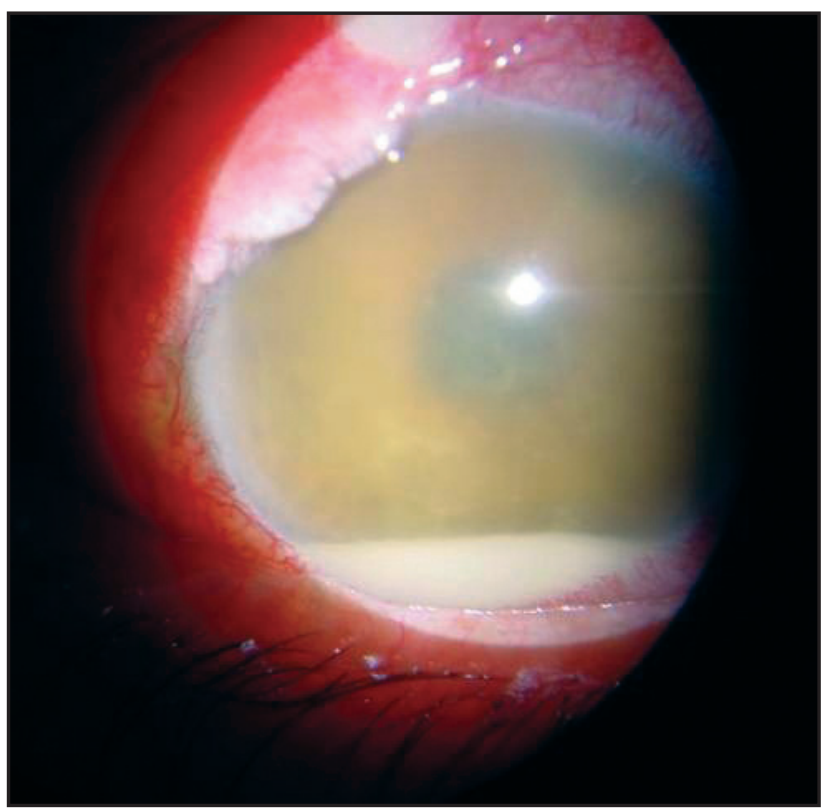

Figura 2: Hipópio e humor aquoso coagulado

mos em $1^{\circ}$. grau. O paciente nasceu de parto natural, a termo, e com boa saúde.

A motilidade ocular era normal, com ortoforia para longe e exoforia para perto. A acuidade visual no OD era 20/100 com $-9,25-2,50$ cil a $90^{\circ}$ e $20 / 20$ no olho esquerdo (OE) com $-0,50$ cil a $120^{\circ}$. Utilizando maleato de timolol $0,5 \%$ colírio nos dois olhos, a PIO aferida foi de $20 \mathrm{mmHg}$ no $\mathrm{OD}$ e $15 \mathrm{mmHg}$ no $\mathrm{OE}$, às 9:30h. $\mathrm{A}$ 


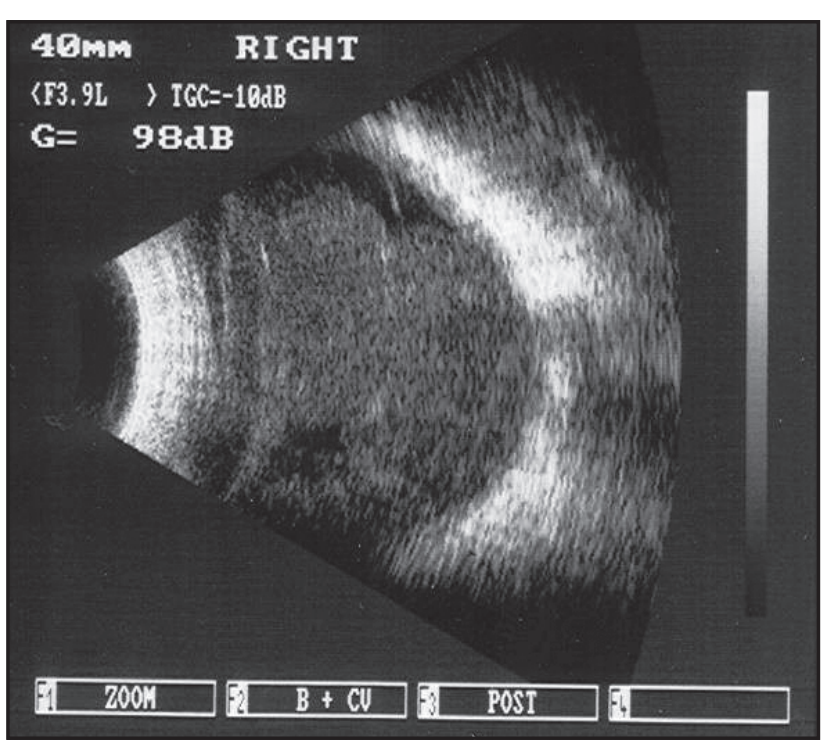

Figura 3: Ecos puntiformes de baixa intensidade ocupando difusa e densamente toda a cavidade vítrea

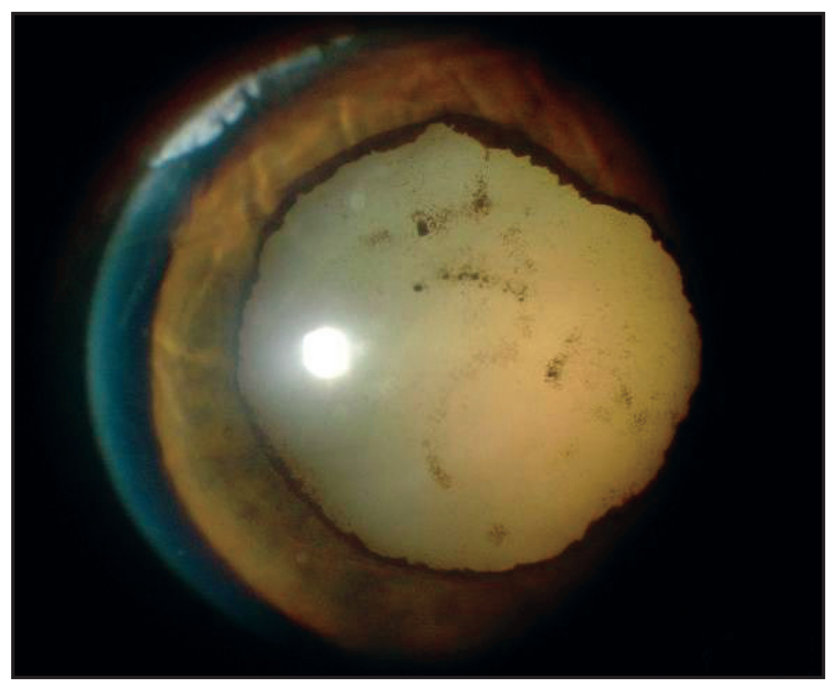

Figura 5: Catarata total intumescente

biomicroscopia mostrou córneas transparentes com megalocórnea no OD, câmara anterior bastante profun$\mathrm{da}$, iridectomia nasal superior em $\mathrm{AO}$ e cristalinos transparentes. A oftalmoscopia mostrou no OD coroidose miópica intensa, atrofia do epitélio pigmentar, cônus miópico e papila atrófica com escavação praticamente total, apenas com estreito remanescente nasal de tecido neural; em OE, a escavação do disco óptico era de 0,6 na vertical $(\mathrm{V})$ x 0,4 na horizontal $(\mathrm{H})$. O diâmetro axial ocular, medido pela técnica de imersão, foi de $30,1 \mathrm{~mm}$ no OD e 24,1 mm no OE. Como a PIO variava de 17 a $23 \mathrm{mmHg}$ no OD na minicurva de pressão intraocular, em abril de 1993 realizou-se trabeculoplastia a laser. No

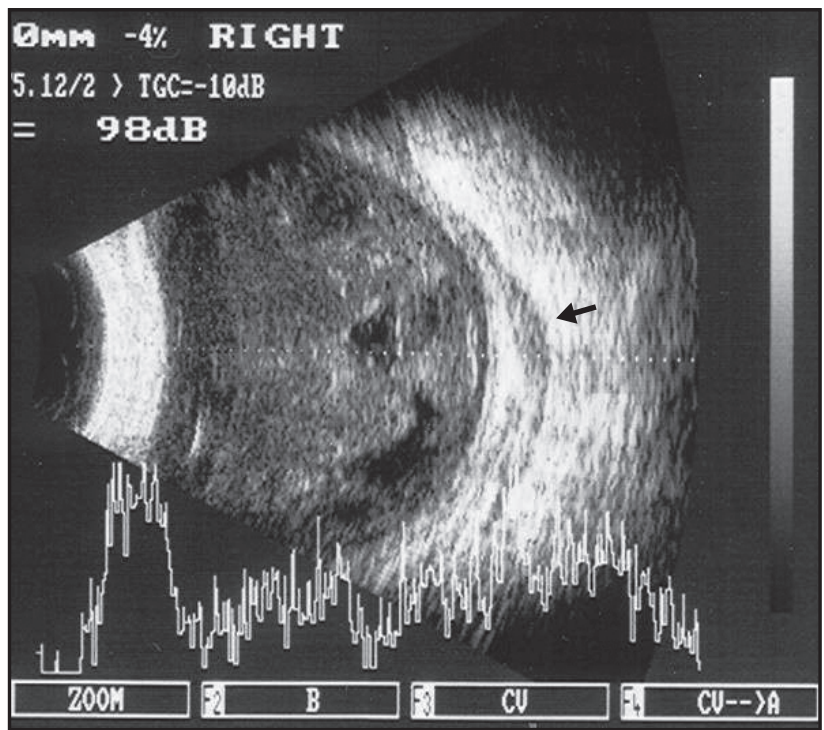

Figura 4: Ecos de baixa e média intensidade na cavidade vítrea preservando lacunas; Edema subtenoniano (seta).

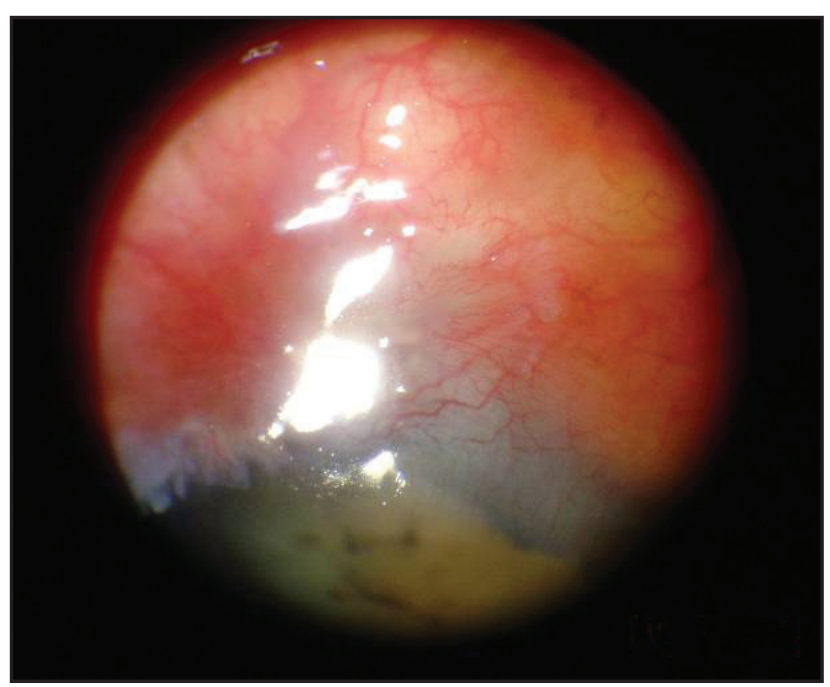

Figura 6: Congestão conjuntival residual e ampola filtrante cicatrizada, sem purulência

OE, a PIO variava entre 12 e $18 \mathrm{mmHg}$.

O paciente somente retornou para novo controle em junho de 1997, em uso de maleato de timolol 0,5\%, quando a PIO no OD era $36 \mathrm{mmHg}$ e no OE $17 \mathrm{mmHg}$. A acuidade visual no OD era 20/80 com -12,00 -1,50 cil a $45^{\circ}$ e no OE $20 / 20 \mathrm{com}+0,50-0,50$ cil a $135^{\circ}$. Como não foi possível controlar a PIO com tratamento clínico, foi realizada em 17/09/97 uma trabeculectomia superior (12h) com retalho conjuntival de base fórnice e mitomicina $\mathrm{C} 0,3 \mathrm{mg} / \mathrm{ml}$ durante 3 minutos no $\mathrm{OD}$, que foi lavada após este tempo. A PIO permaneceu entre 10 e $13 \mathrm{mmHg}$ no OD, sem medicação. No OE, a PIO oscilava entre 17 e $18 \mathrm{mmHg}$ com maleato de timolol 0,5\% 
12/12h. Foi então introduzido cloridrato de dorzolamida $2 \%, 12 / 12 \mathrm{~h}$ neste olho. Somente há referência a campimetria computadorizada em fevereiro de 1999, demonstrando em OD escotoma arqueado superior conectado à mancha cega aberto para a periferia $\mathrm{e}$ escotoma arqueado no quadrante nasal inferior no OE. Em junho de 2000, como a PIO no OE era $18 \mathrm{mmHg}$ com maleato de timolol $0,5 \%$ + cloridrato de dorzolamida $2 \%$, foi acrescentado tartarato de brimonidina $0,2 \%$, todos de $12 / 12 \mathrm{~h}$.

No dia 26 de abril de 2005, o paciente foi atendido pela primeira vez neste Serviço com dor ocular no OD, congestão conjuntival, acuidade visual igual a percepção luminosa (PL), quemose conjuntival, infiltração purulenta da ampola filtrante (figura 1), sem seidel, hipópio (figura 2) com humor aquoso coagulado e duas iridectomias, às 10 e $12 \mathrm{~h}$. Não era possível visualizar o cristalino nem o fundo de olho. A gonioscopia foi inviável no OD, enquanto que no OE o seio camerular se mostrava moderadamente aberto (visualizado até esporão escleral) com periferia da íris mais convexa e pigmentação dentro da normalidade. O OE mantinha acuidade visual $(\mathrm{AV})=20 / 20$ com correção e se mostrava calmo com conjuntiva superior fibrosada, sem ampola filtrante, iridectomia às $11 \mathrm{~h}$, cristalino transparente e fundo de olho normal, com escavação papilar 0,5. A PIO era 12 $\mathrm{mmHg}$ no $\mathrm{OD}$ e $16 \mathrm{mmHg}$ no $\mathrm{OE}$, às 8:40h. Utilizava no OD acetato de prednisolona $1 \% 3 / 3 \mathrm{~h}+$ moxifloxacino $0,5 \% 1 / 1 \mathrm{~h}+$ atropina $1 \%, 6 / 6 \mathrm{~h}$.

A ecografia B mostrou a cavidade vítrea totalmente tomada por ecos puntiformes de baixa e média intensidade (figuras 3 e 4), preservando algumas lacunas (figura 4), ausência de membranas e edema subtenoniano (figura 4, seta). Chamava atenção o significativo aumento das dimensões do OD (figura 3) em relação ao OE (figura 5).

A cultura do aspirado vítreo foi negativa e a coloração pelo Gram mostrou apenas piócitos. Foi realizada uma injeção intravítrea de ceftazidima $(1 \mathrm{mg} / 0,1 \mathrm{ml})$ + vancomicina $(1 \mathrm{mg} / 0,1 \mathrm{ml})+$ dexametasona $(0,4 \mathrm{mg} /$ $0,1 \mathrm{ml}$ ), perfazendo um total de $0,3 \mathrm{ml}$, mantida a medicação tópica de que o paciente já fazia uso e associado a prednisona oral numa dose inicial de $60 \mathrm{mg} / \mathrm{dia}$ com desmame ao longo de 2 meses. Houve regressão completa das manifestações inflamatórias com preservação anatômica do olho, porém com perda completa da acuidade visual e hipotrofia.

Em seu último exame, no dia 07 de julho de 2005, quando ainda fazia uso da medicação tópica , a PIO no OD era 0 (zero) com ausência de percepção luminosa. O olho se apresentava hipotrófico (enoftálmico), com

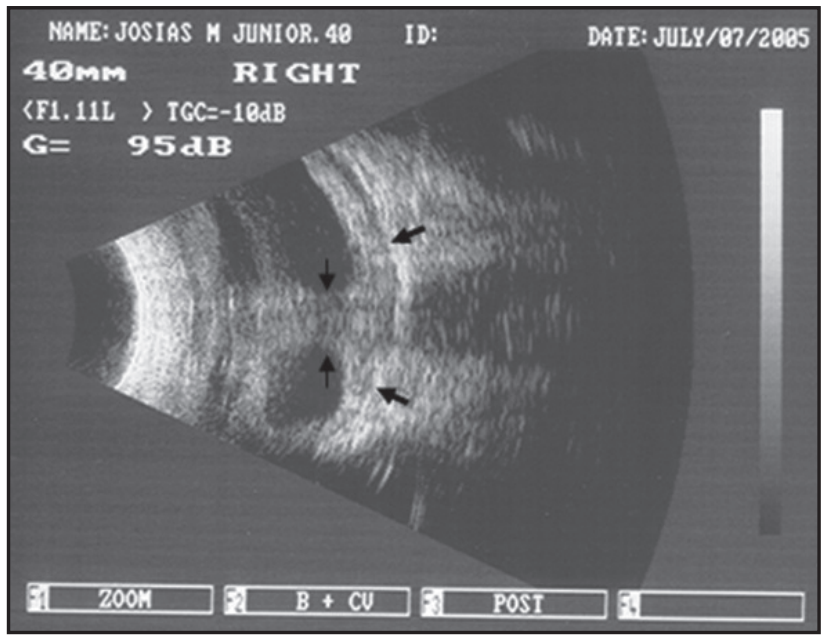

Figura 7: OD hipotrófico (compare com a figura 8) com difuso e marcante espessamento coroidiano (setas negras) e descolamento total da retina em funil fechado (setas negras verticais)

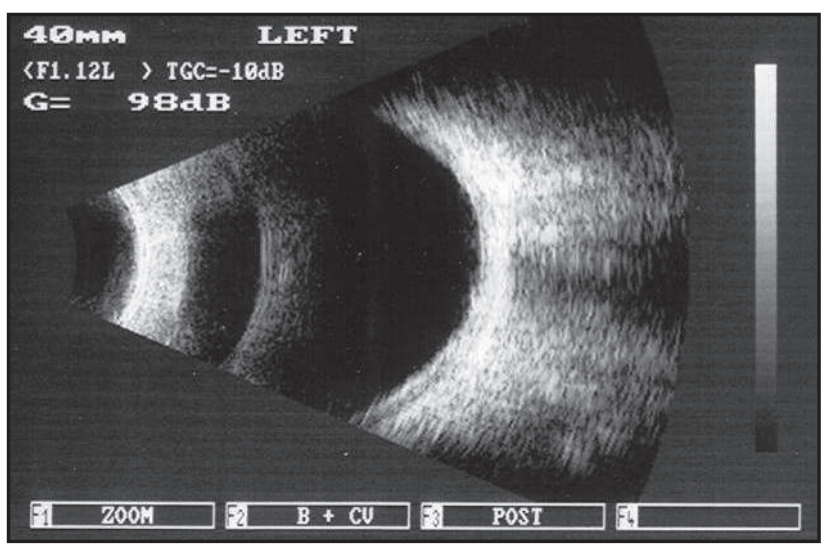

Figura 8: OE com ecografia B normal e dimensões preservadas

congestão conjuntival residual, catarata total intumescente (figura 6), sínfise íridocristaliniana e câmara anterior muito profunda, em pneu. Não havia mais purulência na região da ampola filtrante, que se mostrava cicatrizada e plana (figura 7).

A ecografia B (figura 8) mostrava um quadro de hipotrofia ocular com notável diminuição dos diâmetros oculares em relação ao OE (figura 5), marcante espessamento de toda a coróide (setas negras) e descolamento total da retina em funil fechado (setas negras verticais).

\section{Discussão}

A incidência de infecções relacionadas à presença de uma ampola filtrante tem aumentado preocupantemente com o uso dos antimitóticos ${ }^{(10,12)}$, dada 
a maior fragilidade em decorrência de sua exuberância, avascularidade e fragilidade parietal, que a tornam mais suscetível a vazamentos $^{(7-11)}$ e infecções ${ }^{(13)}$. A infecção, numa fase inicial, se restringe à ampola filtrante, o que é conhecido na literatura anglo-saxônica como "blebitis", para, depois, se estender à câmara anterior (endoftalmite) e, por fim, envolver também o segmento posterior e, até mesmo, estruturas orbitárias, configurando uma panoftalmite. Obviamente, estas são fases de gravidade crescente de um mesmo processo, com implicações diagnósticas, terapêuticas e prognósticas completamente distintas. A primeira dificuldade quando se estuda este assunto na literatura é a falta de critério dos autores no que concerne a estes conceitos e os critérios de exclusão e inclusão das amostragens selecionadas. Alguns autores deixam claro que o envolvimento do vítreo não foi pré-requisito para o diagnóstico de endoftalmite, o que para outros era indispensável ${ }^{(11,12,14)}$, inclusive com comprovação ecográfica ${ }^{(15)}$. Tais casos, então, deveriam ser caracterizados como portadores de panoftalmite, como o caso apresentado, e não como endoftalmite. Mais comumente, estas infecções são tardias, podendo ocorrer décadas após a cirurgia, como neste caso, deflagrado 8 anos depois.

Dentre os fatores de risco para esta complicação cirúrgica após uma trabeculectomia, estavam presentes neste caso o uso de mitomicina $\mathrm{C}$, o fato do paciente ser agricultor, o que, certamente, o expunha mais a fontes potenciais de contaminação, e seu baixo nível sócio-cultural, o que pode condicionar hábitos higiênicos que aumentem sua suscetibilidade neste sentido. Não foi observada a presença de vazamento no transcurso do tratamento e não se dispunha de informações sobre as características da ampola filtrante imediatamente antes do quadro infeccioso. Em um estudo sobre antibioticoterapia profilática em olhos com ampolas filtrantes, não foi observada nenhuma diferença significativa na flora conjuntival dos olhos tratados a longo prazo em relação aos não tratados e não houve garantia de que uma endoftalmite não se desenvolvesse ${ }^{(16)}$.

Nos casos de infecção restrita à ampola filtrante, a cultura do seu material pode ser positiva em até $92 \%$ dos olhos. O índice de cultura conjuntival positiva parece ser mais baixo (64,3\%). Quando há o envolvimento da cavidade vítrea, a cultura do aspirado vítreo é positiva na maioria dos casos $(83,0 \%, 77,0 \%, 83,3 \%)^{(15)}$, mas em alguns trabalhos foi significativa a ocorrência de culturas negativas $(45,0 \%, 30,8 \%)^{(14)}$, o que se deu também no caso apresentado. Tem sido referido na literatura que olhos com cultura negativa tendem a ter um prognóstico visual melhor do que olhos infectados. Os agen- tes mais comuns nas infecções tardias relacionadas a uma ampola filtrante são Streptococcus faecalis, Staphilococcus aureus, Pneumococcus, Enterococcus e Hemophilus influenzae e nas precoces, Streptococcus faecalis, Staphilococcus epidermidis e aureus ${ }^{(16)}$.

$\mathrm{O}$ tratamento clínico das infecções restritas à ampola filtrante ou apenas com envolvimento do segmento anterior deve ser somente local, com colírios: fluorquinolonas de última geração ou fortificados de amicacina $(20-50 \mathrm{mg} / \mathrm{ml})+$ vancomicina $(50 \mathrm{mg} / \mathrm{ml}) \mathrm{de}$ $2 / 2 \mathrm{~h}$ alternadamente + corticosteróides + atropina, associados a injeções subconjutivais de vancomicina $(25 \mathrm{mg} /$ $0,5 \mathrm{ml})+$ ceftazidima $(400 \mathrm{mg})+$ dexametasona $(4 \mathrm{mg})^{17}$. Se o processo ainda estiver restrito unicamente à ampola filtrante, às vezes, apenas os colírios podem ser suficientes e o tratamento pode ser feito em regime ambulatorial com sucesso. Se há envolvimento do vítreo, deve-se, também, optar pela injeção intravítrea de vancomicina (1 $\mathrm{mg} / 0,1 \mathrm{ml})+$ amicacina $(400 \mu \mathrm{g} / 0,1 \mathrm{ml})$ ou ceftazidima $(2,25 \mathrm{mg} / 0,1 \mathrm{ml})$ ou gentamicina $(0,1 \mathrm{mg} / \mathrm{ml})+$ dexametasona $(0,4 \mathrm{mg} / 0,1 \mathrm{ml})$, como no caso presente, e, eventualmente, também TPA 12,5 $\mu$ g. Não está claro se a antibioticoterapia sistêmica nas endoftalmites é benéfica; além do mais, o seu não uso evita efeitos tóxicos, custos e hospitalização ou o seu prolongamento ${ }^{(17)}$.

A indicação de vitrectomia nas endoftalmites ainda é muito controversa. Para alguns autores, é um imperativo uma vez constatado o envolvimento do vítreo, mas segundo o "Endophthalmitis Vitrectomy Study"(17) não é necessária em pacientes com visão melhor do que percepção luminosa, mas é de substancial benefício para aqueles com visão apenas de percepção luminosa. Contudo, alguns autores ponderam com muita propriedade que os resultados deste estudo não devem ser extrapolados para as infecções relacionadas à presença de uma ampola filtrante, dada a patogênese distinta e o espectro único e diverso dos agentes etiológicos. Um estudo não muito recente mostrou que pacientes submetidos à vitrectomia podem ter melhor evolução ${ }^{(18)}$. Por falta de consenso neste sentido, este procedimento não foi realizado neste caso.

$\mathrm{O}$ quadro final de hipotrofia com descolamento total da retina, amaurose e catarata total deixam claro a gravidade desta complicação após uma trabeculectomia com mitomicina $\mathrm{C}$ e sua ocorrência tardia é como uma espada de Dâmocles sobre a cabeça destes pacientes, para sempre. Embora a trabeculectomia tenha se tornado mais segura com a técnica de Peng Khaw ${ }^{(19)}$ e o uso da mitomicina seja rotina na maioria dos serviços, é mister ser parcimonioso no uso desta droga, bem como esclarecer aos pacientes no sentido de se cercarem de medidas 
preventivas e buscarem imediata assistência médica aos menores sinais ou sintomas desta terrível e desastrosa complicação relacionada à presença de uma ampola filtrante! Talvez neste caso, o nível sócioeconômico e a atividade profissional do paciente tenham sido fundamentais no desenvolvimento da infecção ocular.

\section{Abstract}

An intraocular infection is the most terrible complication after a trabeculectomy, usually with significant anatomical consequences and irreversible functional impairment. The case of a 40-year-old male patient with congenital glaucoma, submitted to a trabeculectomy on both eyes at the age of 13, is presented. At the age of 32 a second trabeculectomy with mitomycin $C$ was performed on the right eye. Eight years later he presented with an endophthalmitis in the same eye. Despite intensive local treatment and intravitreous injection, a hypotrophic amaurotic eye resulted with total cataract and retinal detachment.

Keywords: Panophthalmitis; Trabeculectomy; Mitomycin; Antibacterial agents; Case reports

\section{ReFERÊNCIAS}

1. Greenfield DS, Suñer IJ, Miller MP, Kangas TA, Palmberg PF, Flynn WF Jr. Endophthalmitis after filtering surgery with Mitomycin. Arch Ophthalmol. 1996;114(8):943-9.

2. Sidoti PA, Belmonte SJ, Liebmann JM, Ritch R Trabeculectomy with Mitomycin-C in the treatment of pediatric glaucomas. Ophthalmology. 2000;107(3):422-9.

3. Katz LJ, Cantor LB, Spaeth GL. Early and late bacterial endophthalmitis following glaucoma filtering surgery. Ophthalmology. 1985;92(7):959-63.

4. Wolner B, Liebmann JM, Sassani JW, Ritch R, Speaker M, Marmor M. Late bleb-related endophthalmitis after trabeculectomy with adjunctive 5-Fluorouracil. Ophthalmology. 1991;98(7):1053-60.

5. Mandelbaum S, Forster RK, Gelender H, Culbertson W. Late onset endophthalmitis associated with filtering blebs. Ophthalmology. 1985;92(7):964-72.

6. Caronia RM, Liebmann JM, Friedman R, Cohen H, Ritch R Trabeculectomy at the inferior limbus. Arch Ophthalmol. 1996;114(4):387-91.
7. Soltau JB, Rothman RF, Budenz DL, Greenfield DS, Feuer W. Liebmann JM, Ritch R. Risk factors for glaucoma filtering bleb infections. Arch Ophthalmol. 2000;118(3):338-42.

8. Brown RH, Yang LH, Walker SD, Lynch MG, Martinez LA, Wilson LA. Treatment of bleb infection after glaucoma surgery. Arch Ophthalmol. 1994;112(1):57-61.

9. Chen PP, Gedde SJ, Budenz DL, Parrish RK 2nd. Outpatient treatment of bleb infection. Arch Ophthalmol. 1997;115(9):1124-8.

10. Belyea DA, Dan JA, Stamper RL, Lieberman MF, Spencer WH. Late onset of sequential multifocal bleb leaks after glaucoma filtration surgery with 5-Fluorouracil and Mitomycin C. Am J Ophthalmol. 1997;124(1):40-5.

11. Song A, Scott IU, Flynn HW Jr, Budenz DL. Delayed-onset bleb-associated endophthalmitis. Ophthalmology. 2002;109(5):985-91.

12. Jampel HD, Quigley HA, Kerrigan-Baumrind LA, Melia BM, Friedman D, Barron Y. Glaucoma Surgical Outcomes Study Group. Risk factors for late-onset infection following glaucoma filtration surgery. Arch Ophthalmol. 2001;119(7):1001-8

13. Lehmann OJ, Bunce C, Matheson MM, Maurino V, Khaw PT, Wormald R, Barton K. Risk factors for development of posttrabeculectomy endophthalmitis. $\mathrm{Br} \mathrm{J}$ Ophthalmol. 2000;84(12):1349-53

14. Ciulla TA, Beck AD, Toping TM, Baker AS. Blebitis, early endophthalmitis, and late endophthalmitis after glaucomafiltering surgery. Ophthalmology. 1997;104(6):986-95.

15. Higginbotham EJ, Stevens RK, Musch DC, Karp KO, Lichter PR, Bergstrom TJ, Skuta GL. Bleb-related endophthalmitis after trabeculectomy with Mitomycin C. Ophthalmology. 1996;103(4):650-6.

16. Wand M, Quintiliani R, Robinson A. Antibiotic prophylaxis in eyes with filtration blebs: survey of glaucoma specialists, microbiological study, and recommendations. J Glaucoma. 1995;4(2):103-9.

17. Results of the Endophthalmitis Vitrectomy Study. A randomized trial of immediate vitrectomy and of intravenous antibiotics for the treatment of postoperative bacterial endophtalmitis. Endophthalmitis Vitrectomy Study Group. Arch Ophthalmol. 1995;113(12):1479-96.

18. Busbee BG, Recchia FM, Kaiser R, Nagra P, Rosenblatt B, Pearlman RB. Bleb-associated endophthalmitis. Clinical characteristics and visual outcomes. Ophthalmology. 2004;111(8):1495-503; discussion 1503.

19. Jones E, Clarke J, Khaw PT. Recent advances in trabeculectomy technique. Curr Opin Ophthalmol. 2005;16(2):107-13.

\author{
Endereço para correspondência: \\ Nikias Alves da Silva \\ Rua Júlio Vidal, no 11- apto. 1201 \\ CEP 30310-440 - Anchieta - Belo Horizonte - MG \\ E-mail: nikiasa@hotmail.com
}

